\title{
A Miniature Acoustic Device for Tracking Small Marine Animals or Submerged Drifters
}

\author{
G. FISCHER \\ Department of Electrical, Computer and Biomedical Engineering, University of Rhode Island, Kingston, Rhode Island \\ T. RossBY \\ Graduate School of Oceanography, University of Rhode Island, Kingston, Rhode Island \\ D. MOONAN \\ Department of Electrical, Computer and Biomedical Engineering, University of Rhode Island, Kingston, Rhode Island
}

(Manuscript received 24 July 2017, in final form 26 September 2017)

\begin{abstract}
This paper presents an acoustic archival tag capable of tracking small marine animals. It is also a technology that can be ported to other platforms, such as the next-generation acoustic and Argo floats as well as gliders. Tracking is achieved by standard RAFOS triangulation using the arrival times of unique sound signals emitted by moored sources. At the core of the tag is a custom microchip that controls all system operations. It incorporates the critical acoustic arrival time detector, a thermal sensor, and a pressure sensor interface. All the electronic components are housed inside a cylindrical hydrophone of 25.4-mm length and 10.7-mm diameter. The collected data are archived in nonvolatile memory chips with a total capacity of $4 \mathrm{Mb}$, sufficient storage to record position, temperature, and pressure on an hourly basis for 2 years. The tag consumes $4-5 \mu \mathrm{W}$ in standby mode and between 60 and $90 \mu \mathrm{W}$ while the sound arrival time detector is in operation. The power is provided by two button cell silver-oxide batteries, which enable an active tag lifetime of approximately 2 years.
\end{abstract}

\section{Introduction}

Fish biologists have an urgent need to better understand the life cycle and dynamics of various life forms in the sea and how these depend upon their environment and interact with each other. This is of particular concern to commercially important species such as the wide-ranging tuna at/near the surface; benthic species such as cod, halibut, and flounder; and lobster, which live at the bottom. What are the temporal and spatial scales associated with their life cycles? Where and when are these populations most vulnerable; what might be done to enhance their recruitment, enhance survival, and maximize sustainable fishery production? These are not new questions, of course, and indeed much information has been obtained about their behavioral patterns by means of various kinds of data storage tags (DSTs) that

Corresponding author: Godi Fischer, fischer@uri.edu are attached either externally to the dorsal side of the animal or subcutaneously.

For an individual fitted with a DST, by logging in situ physical parameters-for example, temperature, depth, and light intensity - geographical position is inferred on the basis of retrospective analysis of known hydrographic features of the animal's environment or light level for surface species. Such retrospective positioning is necessarily imprecise because physical features may vary only slightly (horizontally, and vertically in higher latitudes) or be poorly known (at least for purposes of retrospective positioning). These limitations notwithstanding, much has been learned with existing DST designs.

For example, the use of DSTs has been pioneered for nearly a decade in studies of bluefin tuna (e.g., Gunn and Hartog 1999; Block et al. 2001), providing crucial insight into behavior, physiology, and movements. Metcalfe et al. (1994) demonstrated and described selective tidal stream transport in European plaice, a flatfish, in the 
North Sea. While DSTs have provided a lot of original insights, accurate positioning remains elusive for the reasons stated. This limitation has to a significant degree been relaxed thanks to a new technology whereby tiny high-frequency acoustic transmitters, each transmitting a unique identification (ID) code, are attached to fish. These devices are not DSTs in the usual sense, but when a fish tagged with such a transmitter passes within acoustic range of a moored stationary receiver, a record of that event is kept. While fish cannot be tracked continuously, this widely used technology gives valuable insight into the overall range and timing of their movements (Weber 2009; Gazit et al. 2013).

In this paper we describe the development of state-ofthe-art technology to track fish continuously over a wide range of distances. Two technologies make this development possible. The first is an acoustic navigation concept in the ocean known as RAFOS, and the second is cost-effective access to customer-design microchip technology. The first, RAFOS, is widely used to determine the trajectories of free-drifting subsurface floats (RAFOS floats), the movements of which can be determined from the arrival times of specially programmed acoustic signals transmitted from distant sound sources. Unlike the older SOFAR float system (see section 2), the reversed role of transmitter and receiver in the RAFOS float system makes it possible to deploy just a few stationary sound sources as navigation beacons, whose very low-frequency transmissions, centered at $260 \mathrm{~Hz}$, can be heard at $10^{2}-10^{3} \mathrm{~km}$ distances depending on acoustic propagation conditions and source power (Rossby et al. 1993). The peak sound pressure level generated by a RAFOS source is close to $180 \mathrm{~dB}$ re $\mu \mathrm{Pa}$ at $1 \mathrm{~m}$. This value is on par with the pressure level generated by some whales and is not expected to harm marine life (Parvin et al. 2007). The second technology, customer-design microchip technology, allows for the development of electronic chips designed to provide specific functionality, in our case an acoustic receiver and datalogger that can operate at extremely low power for several years.

\section{Background}

A truly remarkable property of the ocean is its acoustic transparency (Urick 1984). This property has been used for many purposes, such as echo sounding, fish localization, antisubmarine warfare, acoustic thermometry of the oceans, and the tracking of subsurface drifters over great distances. The existence of the deep sound channel or the SOFAR channel has allowed for the localization of sound sources at distances well in excess of $1000 \mathrm{~km}$. Oceanographers have used floats extensively to trace the movement of water parcels as they are carried by ocean currents (e.g., Bower et al. 1995; Rossby 1996). In the early years, the floats were acoustic transmitters (SOFAR floats; Rossby et al. 1975) with signals that could be picked up at shore-based hydrophones and later on autonomous moorings. With the advent of low-power microprocessors in the late 1970s, it became possible to shrink the entire acoustic receiver and signal processing onto a small circuit board, leading to a much-reduced cost of the floats. The data collected in the RAFOS floats are transmitted back via satellite for analysis at the end of the float's underwater mission (Rossby et al. 1986).

The actual process of detecting the arrival time of an acoustic signal is quite straightforward and is an almost entirely digital process. The traditional SOFAR/ RAFOS float signal consists of a linear increase in frequency from 259.38 to $260.90 \mathrm{~Hz}$ over $80 \mathrm{~s}$. After the incoming signal is amplified, band shifted to zero frequency, and clipped, it consists of a single bitstream reflecting whether it is positive or negative-essentially a one-bit phase digitization. The receiver knows the expected binary sequence, so the remaining step is to compare the incoming 80-s-long bitstream to the expected pattern. This process is known as a cross correlation or an exclusive negated OR gate (XNOR) operation in digital terms. The time at which the best fit occurs is considered to be the time of arrival of that signal. Since all emitted signals are identical regardless of sound source location, their identity is established based on arrival times recorded in previous listening windows combined with the projected float movement. This source identification is carried out retrospectively; the receiver simply stores the arrival times.

The RAFOS technology has been immensely successful; well over 1000 floats have been deployed over the 30-plus years they have been in use. Here we take this technology a step forward by shrinking the entire functionality of the RAFOS float onto a single monolithic chip, which becomes the nerve center of the fish tag. The next section describes the architecture, design considerations, and functionality of the newly developed fish tag.

\section{The fish tag}

\section{a. Tag architecture}

Figure 1 depicts the basic functional components of the tag. At the core of the system is an applicationspecific integrated circuit (ASIC) that performs all control functions, including instructing the tag to execute a specific sampling protocol, calibrating the 


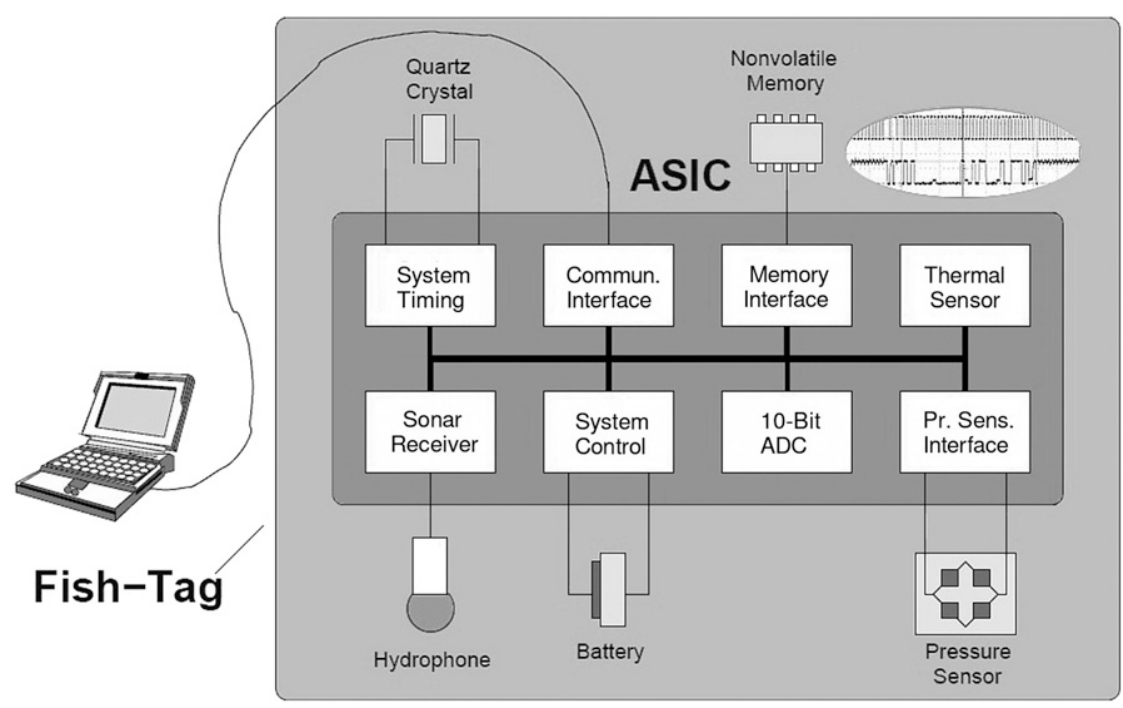

FIG. 1. Basic functional blocks of the fish tag. All components inside the dark gray area are housed on the ASIC.

temperature sensor and the timing unit, and storing the recorded data in external nonvolatile memory chips. The current system can address up to four memory chips with a capacity of $1 \mathrm{Mb}$ each. Upon completion of the calibration and programming phase, the three-wire interface to the host PC is removed so that the tag can be sealed and made ready for deployment. The tag also houses a commercial micromachined pressure sensor to record depth. The $2 \mathrm{~mm} \times 2 \mathrm{~mm}$ sensor provides a range of 300 psi ( $\sim 200-\mathrm{m}$ depth). Because of the potentially large raw sensor offset of as much as one-third of its full range, the pressure sensor interface aboard the ASIC has been configured to accommodate more than twice the nominal sensor range. This reduces the resolution from potentially 0.3 to $0.75 \mathrm{psi}(\sim 0.5-\mathrm{m}$ depth) but eliminates a precalibration procedure. The actual sensor offset and gain values are recorded only after the tag's retrieval. Actually, the offset is known, since the tag is in operation before the fish is released, so it is primarily the gain factor that needs to be determined. Knowing the sensor gain and offset enables a precise digital calibration of the archived pressure values. The resulting accuracy depends, of course, on how well the offset and gain at the actual sampling time match their postretrieval values.

The monolithic thermal sensor makes use of the temperature characteristic of a p-type and n-type $(p-n)$ junction (Vittoz and Neyroud 1979). This solution provides for a small silicon footprint, but similar to the pressure sensor, it suffers from relatively poor device-to-device matching. To mitigate the potentially large temperature offset errors, the sensor has been complemented by a 7-bit digital calibration unit that keeps the maximum raw error below $0.3^{\circ} \mathrm{C}$. Keeping this raw error small is important, because the precalibrated thermal output is used to compensate for the temperatureinduced frequency deviations of the master timing crystal as discussed in section 3d. A postretrieval temperature calibration will reduce the absolute error close to $\pm 0.05^{\circ} \mathrm{C}$.

The remaining two functional components of the tag are the hydrophone and the electric power source. The cylindrical hydrophone, being the largest component, also serves as the housing for all the electronic components. The copolymer hydrophone utilized by the prototype tags is a cylinder of 25.4-mm length with a $10.7-\mathrm{mm}$ outer diameter. Its hydrostatic sensitivity is listed as $-195 \mathrm{~dB}$ re $1 \mathrm{~V} \mu \mathrm{Pa}^{-1}$.

The tag is powered by two series connected silveroxide button cells with a nominal terminal voltage of $1.5 \mathrm{~V}$ and a capacity of $80 \mathrm{mAh}$. With a diameter of only $7.9 \mathrm{~mm}$, the batteries fit tightly inside the hydrophone cylinder. The center tap of the two batteries serves as the common voltage to enable a bipolar voltage swing of $\pm 1.5 \mathrm{~V}$.

The most critical unit of the tag is arguably the sound arrival time detector, in particular the analog receiver section. The next subsection describes this unit in more detail.

\section{b. Acoustic receiver}

By necessity, long-range acoustic tracking requires working at very low frequency somewhere between 200 and $800 \mathrm{~Hz}$ (e.g., Urick 1983). RAFOS and the earlier 
SOFAR floats have very successfully operated at $260 \mathrm{~Hz}$ in a wide variety of environments (Rossby 2007). Regardless of a specific design, most low-frequency sound sources utilize a long pipe to achieve efficient acoustic radiation over a very narrow range of frequencies, typically less than $3 \mathrm{~Hz}$.

The acoustic signature of the fish tag has been chosen different from the traditional RAFOS system to better cope with a more dynamic target like a fish versus a float. The frequency modulation range or signal bandwidth has been increased from 1.52 to $3 \mathrm{~Hz}$, and the duration of the signal has been reduced from 80 to $32 \mathrm{~s}$. This renders the tag less vulnerable to Doppler-induced frequency shifts and reduces arrival time or distance errors cause by the relative target motion by a factor of 5 . In addition, the signal center frequency has been slightly increased from 260 to $262 \mathrm{~Hz}$ so that a $256-\mathrm{Hz}$ frequency modulation can shift the signal center to $6 \mathrm{~Hz}$ (Fischer et al. 2006). This allows the final 1-bit digitizer to be clocked at a much lower rate of $32 \mathrm{~Hz}$. This low clock frequency not only saves power but also significantly reduces the size of the digital memory required by the subsequent arrival time detector. The proximity of the fish tag frequency range to the RAFOS transmission makes it possible to use existing RAFOS sources retuned to match the $0.7 \%$ higher transmission frequency.

By implementing the narrowband filter cascade of the fish tag receiver with analog sampled-data circuits (Schneider and Galup-Montoro 2010; Crols and Steyaert 1994), all filter characteristics scale as the sampling rate. This opens up the possibility of reducing the minimum time step of the arrival time detector by simply clocking all sampled-data filter sections at a higher rate. This does, of course, require the acoustic signal to be centered at a correspondingly higher frequency. Altering the filter clocks can be readily achieved by complementing the crystal oscillator with a low-power phase-locked loop designed to generate a range of integer fractions (e.g., $n / 4$, $n=\{5,6,7,8\}$ ) of the original crystal frequency (Fischer and Luo 2012).

Figure 2 shows a measured spectral response of the acoustic receiver. The plot has been generated via of a 1-million-point FFT using a sampling rate of $10 \mathrm{kHz}$. To obtain this $100-$ s-long recording, a $262-\mathrm{Hz}$ sinusoid of 500-nV amplitude was directly fed into the first stage of the filter cascade. The data were collected by a digital oscilloscope. The amplified signal component is clearly visible in the spectral plot. The power of the displayed in-band noise-that is, the sum of all spectral components between 4.5 and $7.5 \mathrm{~Hz}$ with the exception of the $262-\mathrm{Hz}$ signal-amounts to $-42.8 \mathrm{~dB}$. This corresponds to an equivalent noise voltage of $7.2 \mathrm{mV}$. Dividing this voltage by the nominal receiver gain of $18000(85 \mathrm{~dB})$

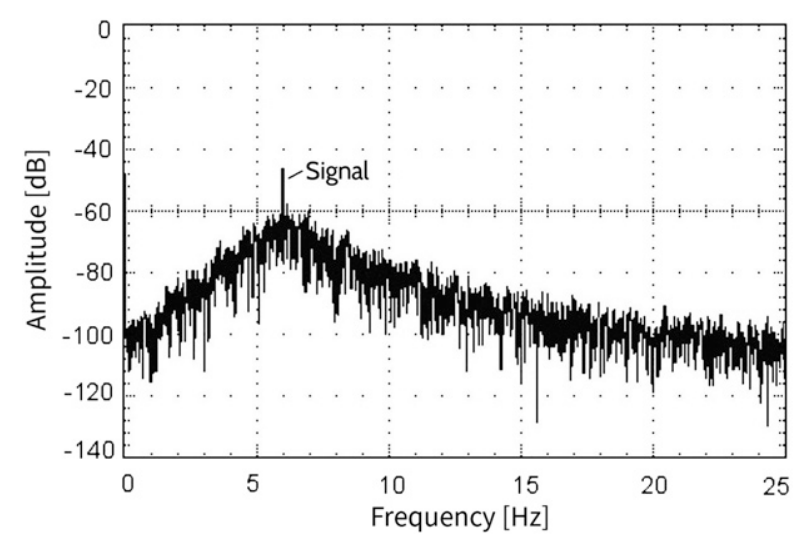

FIG. 2. Spectral response of a receiver filter cascade in the presence of a $262-\mathrm{Hz}$ sinusoidal input of 500-nV amplitude. The spectrum has been computed via a 1-million-point FFT using a sampling rate of $10 \mathrm{kHz}$. This yields a spectral resolution of $0.01 \mathrm{~Hz}$.

yields an equivalent input referred noise voltage of $400 \mathrm{nV}$. The ambient noise voltage picked up by the copolymer hydrophone with a sensitivity of $-195 \mathrm{~dB}$ re $1 \mathrm{~V} \mu \mathrm{Pa}^{-1}$ is expected to be somewhere between $400 \mathrm{nV}$ and $4 \mu \mathrm{V}$ depending on sea state and other environmental conditions (Wenz 1962; Urick 1984; Harrison 1996). The receiver self-noise is therefore not expected to limit the detection range.

\section{c. Arrival time detector}

To find a reliable estimate for the arrival time of a specific sound signature-in this case, a narrowband linear frequency sweep-the detector compares the digital output stream of the acoustic receiver to a stored replica of the expected signature. The time corresponding to a maximum overlap between the two signals constitutes the best estimate for the arrival time. By quantizing the signal at the end of the receiver cascade to 1 bit, this comparison can be carried out by a single logic XNOR as stated in section 2. The output bitstream of this gate is then fed into a binary counter that accumulates the number of matched pairs over a length of 1024 samples. To accomplish this in real time, the counter needs to be clocked 1024 times per output period. The nominal output rate of $32 \mathrm{~Hz}$ thus requires a correlator clock rate of $32.768 \mathrm{kHz}$. This value is particularly convenient, since it is the clock frequency used by almost all electronic watches. Watch crystals are widely available and inexpensive.

Figure 3 illustrates the efficacy of the described crosscorrelation procedure. Since the microchip does not allow for direct observation of the correlator function, we resorted to a simulation to illustrate the applied procedure. The top-left window in Fig. 3 shows an ideal 

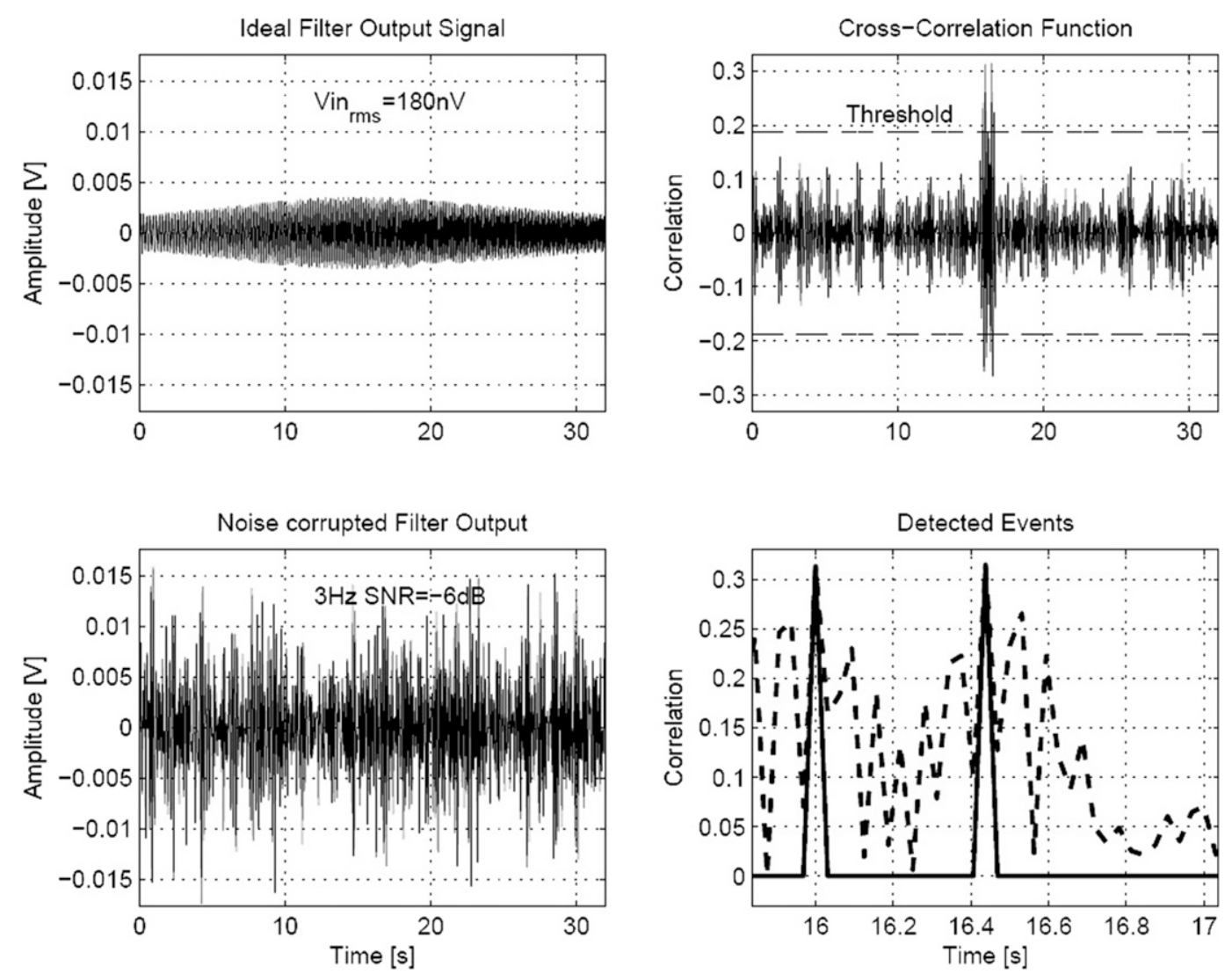

FIG. 3. (top left) The response of the receiver to an ideal rms input voltage of $180 \mathrm{nV}$. (bottom left) A scenario with two 32-s-long input signals spaced $0.4 \mathrm{~s}$ apart in time. Both inputs feature an rms voltage of $180 \mathrm{nV}$ and an equivalent noise component of $360 \mathrm{nV}$, representing an SNR of $-6 \mathrm{~dB}$. (top right) The corresponding correlator function, and (bottom right) the two signal arrival time estimates deduced by the peak detector.

receiver output over a full signature length of $32 \mathrm{~s}$ or 1024 samples. The window below represents a more challenging scenario with two acoustic signatures arriving just $0.4 \mathrm{~s}$ apart (a $98.8 \%$ overlap in time) and deeply embedded in noise. In fact, the depicted in-band noise power exceeds each signal power by a factor of 4 . This corresponds to a signal-to-noise ratio (SNR) of $-6 \mathrm{~dB}$. The top-right window depicts the output of the digital cross correlator. To avoid filling the four available memory slots per acoustic listening window with correlation peaks pertaining to the same event and so preventing the detection of weaker signatures, a peak detector has been added that records only the maximum of 16 consecutive correlation values. This 16 -sample or 0.5 -s-long window is triggered by any correlation value larger than 0.1875 . Lower values are disregarded, since they are not very different from random correlation spikes caused by the stochastic receiver output noise. The stated threshold corresponds to 608 matches out of $1024(59.4 \%)$. Since the peak detection operates in parallel with the correlation counter, it does not skip any correlation values and thus provides a continuous record of all relevant peaks in the correlator function. The bottom-right window in Fig. 3 reveals that the simulated peak detector identified two distinct maxima separated by $0.4375 \mathrm{~s}$. This is one sampling period more than the actual signature separation of $0.4 \mathrm{~s}$. The fact that each maximum in the bottom-right plot is flanked by a number of prominent side peaks underlines the need for a peak detector.

Correctly identifying and timing the relevant correlation peaks does not necessarily lead to a correct geographic position. Doppler frequency shifts caused by the relative velocity between source and receiver can move a fraction of the signal spectrum out of band and shift the arrival time estimate accordingly. Even though the fish tag receiver is 5 times less Doppler sensitive than the RAFOS detector, $1 \mathrm{kt}\left(0.51 \mathrm{~m} \mathrm{~s}^{-1}\right)$ of relative motion still causes a time-of-arrival error of $0.9 \mathrm{~s}$, which translates into a distance error of 0.75 nautical miles ( $\mathrm{n} \mathrm{mi}$; $1 \mathrm{n} \mathrm{mi}=1.852 \mathrm{~km}$ ). Furthermore, if the relative source versus receiver velocity varies noticeably over the 32 -s 


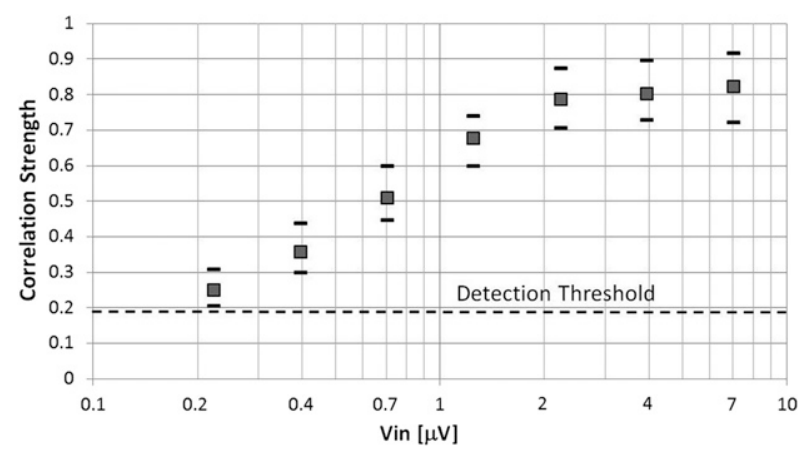

FIG. 4. Recorded correlation strength vs receiver input voltage. The plot marks the minimum, maximum, and mean of 12 consecutive recordings conducted per input signal level.

signature duration, the sound signature will be distorted, which in turn reduces the number of matched pairs registered by the correlation counter. Both of these effects scale as the inverse signal bandwidth. Another reason for diminished correlation peaks is the random time or phase shift between received and stored signal patterns. This alignment error is due to the discrete time nature of the output signal and scales as the receiver sampling period.

Figure 4 illustrates the relationship between correlation strength and signal swing. The plot shows the minima, mean, and maxima of 12 recorded correlation values per applied signal swing. To be able to precisely control the input level, we again fed the output of a signal generator directly to the preamplifier input of the analog receiver. Intersecting the line of mean correlation peaks in Fig. 4 with the detection threshold (dashed line) reveals a receiver sensitivity of approximately $180 \mathrm{nV}$. This is 2.2 times or $7 \mathrm{~dB}$ less than the equivalent receiver input noise voltage. The observed correlation ceiling near a value of 0.8 is due to the aforementioned random alignment error between the actually received signature and the stored replica.

\section{d. The tag clock}

The key to most electronic tracking systems, including GPS, is accurate timing-more specifically, accurately recording the arrival times of characteristic patterns emitted by multiple sources at known locations. Knowing the signal propagation speed and the travel times of all detected signals, one can infer the position of the receiver via triangulation (Eriksson et al. 1994). To obtain the most accurate estimate for position, source and receiver clocks have to be perfectly synchronized. Keeping source and receiver synchronized over long time spans without periodic corrections, however, remains a serious issue: a static clock error of only $1 \mathrm{ppm}$ accumulates to a deviation of $32 \mathrm{~s}$ over one year.

Since the fish tag is severely limited in energy capacity, it employs a commercial $32.768-\mathrm{kHz}$ watch crystal oscillator with a current drain of just 300-500 nA. This crystal oscillator is manufactured for room temperature operation and thus features a zero temperature gradient near $25^{\circ} \mathrm{C}$ rather than $10^{\circ}-12^{\circ} \mathrm{C}$, which would be more desirable for underwater applications. In addition, its nominal frequency can deviate by as much as $\pm 20 \mathrm{ppm}$ as a result of variations in the production process. To counter this, the fish tag timer employs a digital correction unit that allows adjusting for offset errors and compensating for the expected temperature deviations. The 6-bit digital correction scheme consists of two parts, a static correction to eliminate the offset caused by fabrication process variations and a dynamic portion to compensate for the temperature-induced frequency deviations. Compensating for a possible worst-case error of $\pm 45 \mathrm{ppm}$ (static plus dynamic) requires a minimum correction step of $1.4 \mathrm{ppm}$. We have selected $1.9 \mathrm{ppm}$, since this corresponds to adding or subtracting one clock cycle in $2^{19}$, which, for the $32.768-\mathrm{kHz}$ clock, equates to one correction per $16 \mathrm{~s}$ so that the residual errors are equally distributed between $\pm 0.95 \mathrm{ppm}$. Figure 5 graphically illustrates the applied digital correction scheme.

In a dynamic environment, the cumulative timing error should approach zero, since each temperature causes a different error uniformly distributed between the two bounds of $\pm 0.95 \mathrm{ppm}$. Realistically, each oscillator will deviate slightly from its expected temperature characteristic, leaving a residual cumulative error. Furthermore, in a scenario where the temperature remains constant over long periods of time, cumulative errors can become quite prominent. This scenario could arise in deep-water fish studies, where the temperature is rather stable. But there are two ways clock errors can be reduced retroactively. First, when a tag is recovered, the measured clock error coupled with a postcalibration of the crystal's temperature dependence can lead to an improved clock error history. The second approach, which is often used in RAFOS, uses travel time differences from three sound sources to determine position hyperbolically. Knowing position, one can then retroactively determine the clock correction needed to obtain the right travel times (Blanchard 1991).

To validate the expected behavior of the cumulative timing error, we recorded temperature and acoustic arrival times with two of our prototype tags over a period of 10 days. Temperature was recorded hourly, but the acoustic stimulus was applied only once a day. To obtain 


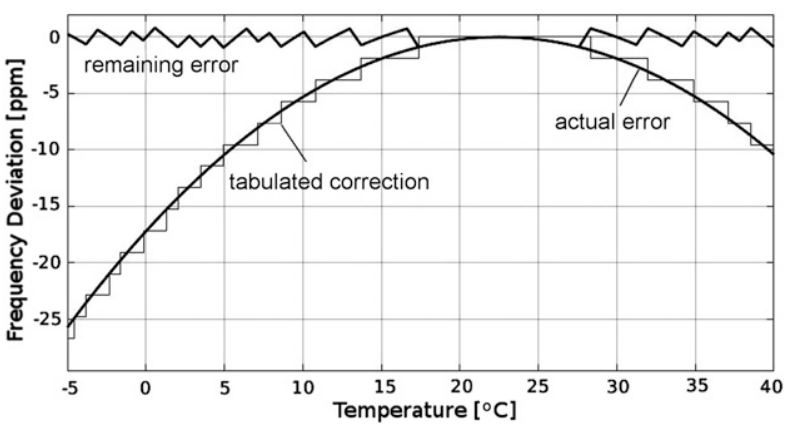

FIG. 5. Illustration of applied digital correction scheme mitigating the temperature-induced frequency deviations of the watch crystal.

different temperature profiles, we kept one tag at room temperature and the other one was intermittently exposed to a colder environment.

The top plot in Fig. 6 displays the two recorded temperature profiles, while the bottom one reveals the corresponding cumulative timing errors. In spite of the two distinctly different temperature records, the arrival time errors recorded during this 10-day test stayed within bounds of $\pm 1 \mathrm{ppm}$. Without the digital clock correction, the cumulative timing error of either tag would likely have amounted to several seconds over the 10-day test period.

Figure 7 presents a micrograph of the core microchip. This $1.5 \mathrm{~mm} \times 1.5 \mathrm{~mm}$ die, referred to as a fish chip, accommodates all basic functional units of the tag, including the sound arrival time detector, the temperature sensor, the pressure sensor interface, the 10-bit dual-slope analog-to-digital converter, the clock tuning circuitry, and the central controller responsible for the timely execution of all sampling tasks and data transfers to and from the tag. To minimize the crosstalk between analog and digital building blocks, all analog functions have been realized inside a relatively narrow column on the right side of the depicted die, surrounded by protective guard rings. Most of the remaining chip area is filled by two identical digital correlator circuits and a common random-access memory (RAM) that archives the selected sound signature (Pattern RAM) and temporarily stores the incoming bitstream generated by the receiver (Hydro RAM). By simultaneously reading the Pattern RAM in forward and backward modes and feeding each data stream to a designated correlator, the chip executes two concurrent signal arrival time detections. This two-way discrimination does not require physically different sound sources; they simply have to be instructed to emit the selected signal pattern in forward and time-reversed modes, respectively. A potential benefit of employing both ascending and descending frequency modulation is that the Doppler shift will be in
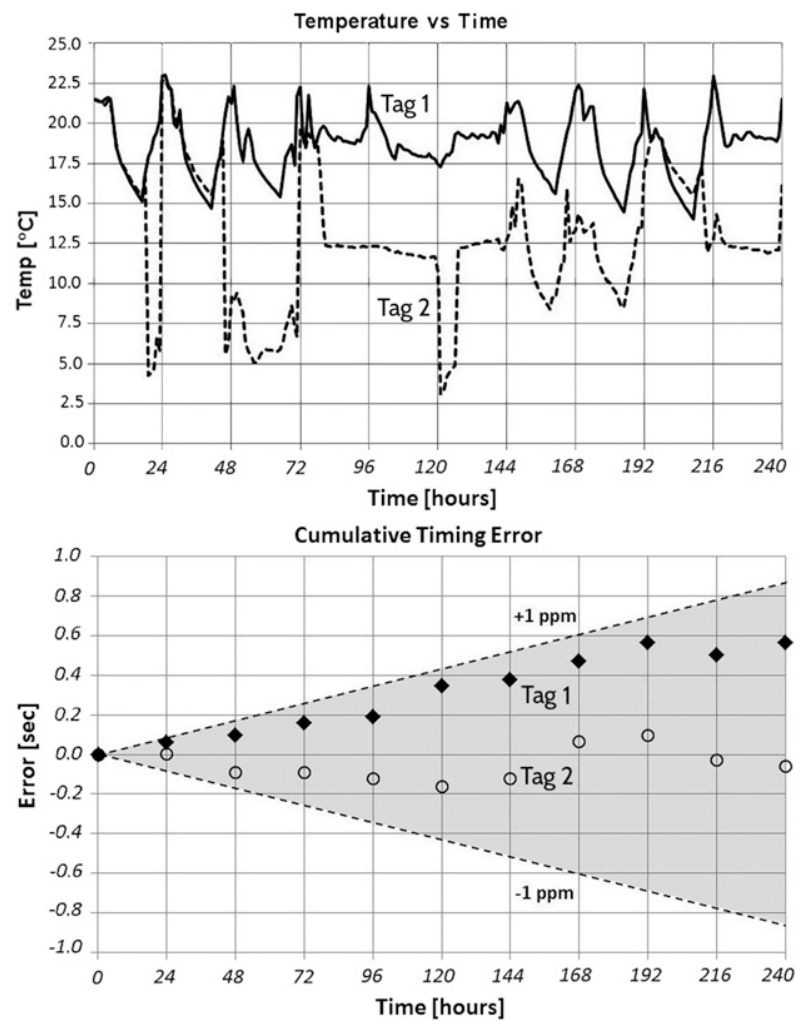

FIG. 6. (top) Temperature recordings during a 10-day timing test, and (bottom) the corresponding cumulative clock error assessed once a day.

opposite directions. The average time of arrival will remove the Doppler effect, while the difference between the two will provide a measure of fish tag speed in direction of the sound source.

\section{e. Tag assembly}

From a conceptual perspective, the tag assembly is a rather straightforward matter. Unfortunately, this statement does not equally apply to the actual physical assembly. The fish chip, housed in a quadrature leadless carrier of just 3-mm side length, is surface mounted on a tiny printed circuit board (PCB) with the other electronic components, that is, the watch crystal oscillator, the nonvolatile memory chips, and three filter capacitors. This PCB is only $8 \mathrm{~mm}$ wide and fits tightly inside the hydrophone cylinder. The circular pressure sensor board is attached perpendicular to the PCB and forms one of the two side plates of the cylindrical tag. The other components of the device are a pair of button cell batteries with connecting wires and the copolymer hydrophone. All components and the final tag are depicted in Fig. 8. The top layer of the tiny PCB on the top left accommodates the custom fish chip and one nonvolatile memory chip. The hidden bottom layer houses a second memory chip, the watch crystal oscillator, 


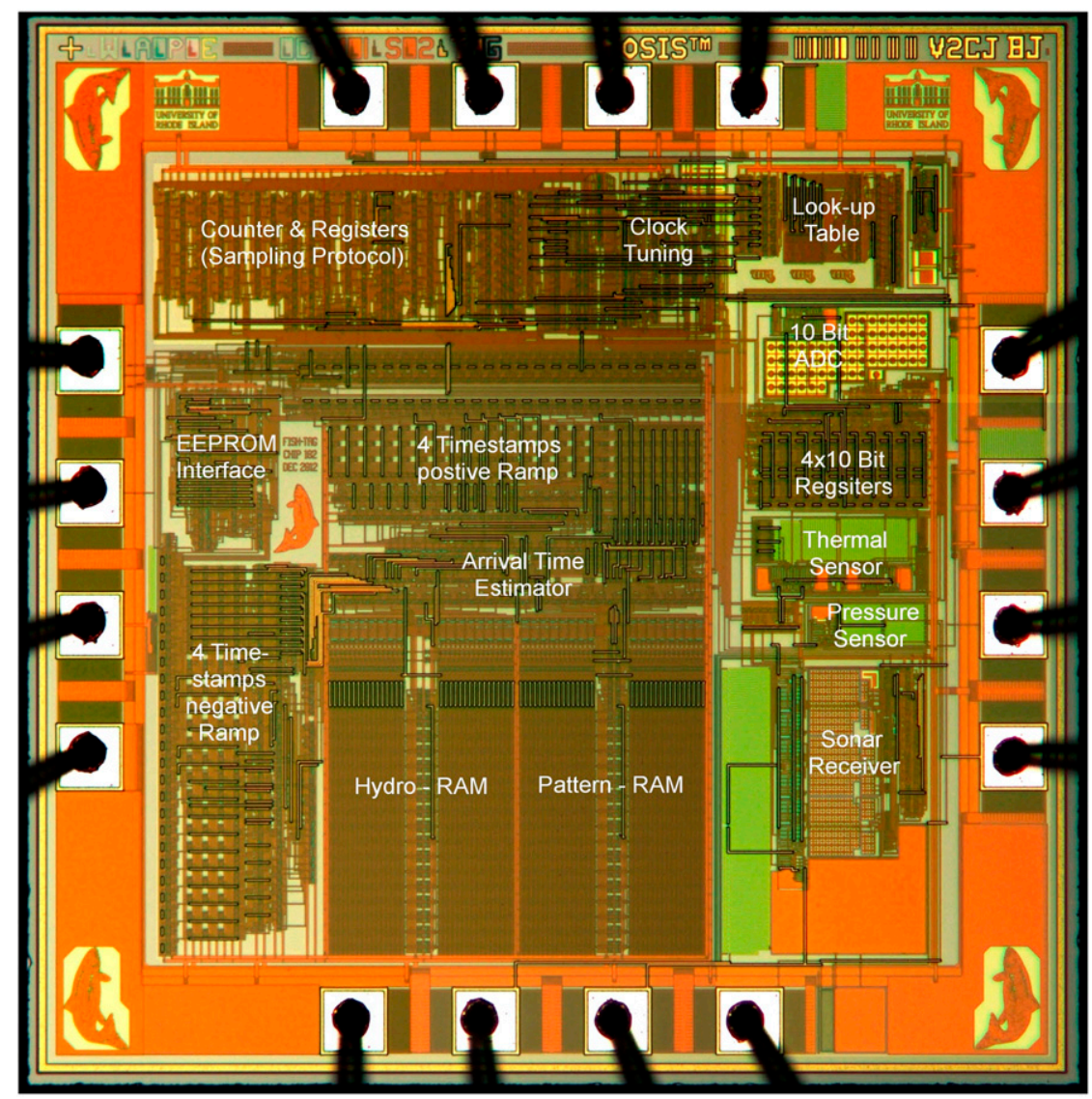

FIG. 7. Micrograph of a fish chip. The annotations reveal the pertinent functional building blocks (die size: $1.5 \mathrm{~mm} \times 1.5 \mathrm{~mm}$ ).

and the three filter capacitors. The fully assembled tag, depicted on the right, is $4.8 \mathrm{~cm}$ long, has a volume of $5.8 \mathrm{~cm}^{3}$, and weighs $8.4 \mathrm{~g}$. Its net weight in seawater is $2.5 \mathrm{~g}$. The component cost for the depicted tag, when purchased for a volume in the low hundreds, is around $\$ 150$. If one adds another $\$ 50$ to include the tag assembly, the production cost amounts to approximately $\$ 200$ per unit, which is roughly $4 \%$ of the cost of a RAFOS float.

\section{f. Sampling protocol and power}

The tag's sampling protocol is defined by four parameters. Temperature, pressure, and battery voltage sampling are determined by two values, the activation time Ta (the time elapsed between programming and start of the sampling mission) and the time Ts between two sensor sampling instances. The remaining two parameters define frequency and length of the acoustic window. Since the acoustic position is likely to be assessed less frequently than temperature, pressure, and battery voltage, the time between two acoustic tracking events has been defined as a power of 2 multiple-that is, $2^{M}$ - of the sensor sampling interval Ts. The user selects the exponent $M$ as a whole number between 0 and 7 . The parameter Tw, finally, indicates the length of the acoustic listening window. Table 1 lists range and step size of each of the three crucial timing parameters: Ta, Ts, and Tw. It also summarizes the capabilities of the three sensors for temperature, pressure, and battery voltage by listing the range and resolution of each.

Prior to each mission, the user uploads the four sampling protocol parameters-Ta, Ts, Tw, and $M$-from a graphical user interface (GUI) on a laptop or tablet. The GUI also asks for two digital corrections, compensating for the thermal sensor offset and the static frequency deviation of the crystal oscillator, respectively. These values have been obtained from the preceding precalibration procedure. Upon completion of the programming phase, the wire-bound interface to the host is removed so that the tag can be sealed and made ready for deployment.

The frequency and duration of the acoustic tracking have a major impact on the power budget. The supply current recorded during active tracking-that is, the time while the tag listens for a sound signature-ranges between 20 and $30 \mu \mathrm{A}$. To illustrate the power usage, let 


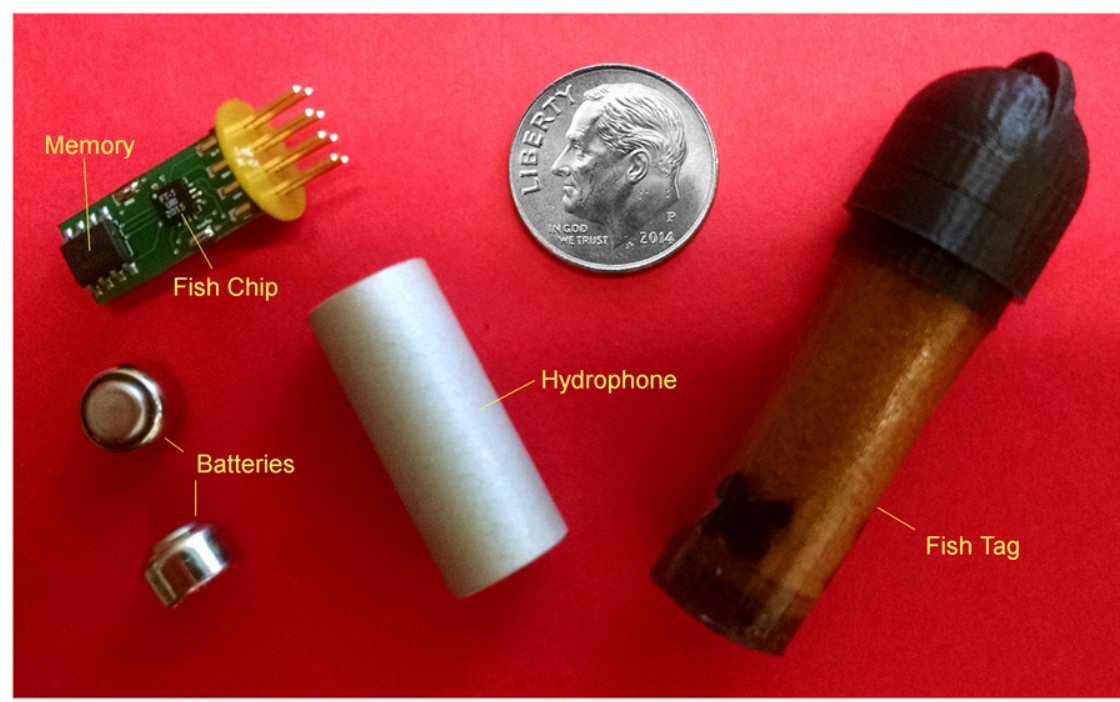

FIG. 8. Fish tag components vis-à-vis a (center top) U.S. dime and (right) an assembled tag.

us assume a scenario where a user programs the tag to assess the geographic position once per hour with an acoustic listening window of $6 \mathrm{~min}$. The additional average current drain then amounts to $2-3 \mu \mathrm{A}$ (1/10 of the above). Selecting the more conservative number and combining it with the observed standby current of $1.5 \mu \mathrm{A}$ yields a total current drain of approximately $4.5 \mu \mathrm{A}$. Combining this average power drain with the $80-\mathrm{mAh}$ charge stored in each of the two series' connected button cell batteries results in an active tag lifetime of $17778 \mathrm{~h}$ or almost exactly 2 years. Assessing the position and sampling temperature, pressure, and battery voltage once an hour for 2 years fills $97 \%$ of the available $4-\mathrm{Mb}$ memory space. Alternatively, recording the position a dozen times per day while sampling temperature, pressure, and battery voltage every $15 \mathrm{~min}$ fills the memory in 682 days but consumes only $56 \%$ of the available battery energy. Since sampling, digitizing, and archiving temperature, pressure, and battery voltage are accomplished in less than $0.2 \mathrm{~s}$, the computational power exerted during these events is practically irrelevant.

\section{Field test results}

To assess the feasibility and to validate the performance of the fish tag under development, a series of field tests have been conducted during the course of this project. The first one was a preliminary test designed to evaluate the critically important analog preamplifier and the potential tracking range. In this experiment, both the source and receiver (the preamplifier and a commercial digital recording device) were positioned below the surface mixed layer at $\sim 40$ and $30 \mathrm{~m}$, respectively, on the
New England shelf south of Nantucket (Fischer et al. 2006). The sound source was operated at the peak power of $180 \mathrm{~dB}$ re $1 \mu \mathrm{Pa}$ at $1 \mathrm{~m}$ for all transmissions. The data collected during this early field experiment showed that the preliminary receiver could repeatedly detect the RAFOS signature at the farthest site $70 \mathrm{~km}$ from the acoustic source. The spectral analysis of the data recorded at the most remote site revealed a surprisingly robust inband signal-to-noise ratio of approximately $6 \mathrm{~dB}$.

A second comprehensive field test took place in September 2015 at a location some $40 \mathrm{~km}$ south of the eastern tip of Long Island. This site provided a relatively uniform water depth of approximately $60 \mathrm{~m}$. At that time, we were not yet ready with final tags as depicted in Fig. 8. Instead, we mounted four fish chips on larger PCBs housed inside four PVC cylinders, each with a volume of $2.2 \mathrm{~L}$. The polyurethane-coated copolymer hydrophones were attached to the bottom plate of each cylinder with wires connected to the fish chip. This assembly provided full thermal and acoustic functionality.

The logistics of the experiment were rather simple. The four PVC cylinders, or pods, were attached to a rope hanging below a free-floating surface drifter. The four pods were placed at depths of $10,20,30$, and $40 \mathrm{~m}$, respectively. The host vessel, the R/V Endeavor, then

TABLE 1. Pertinent fish tag parameters.

\begin{tabular}{ccccccc}
\hline \hline \multicolumn{2}{c}{ Sampling protocol } & & \multicolumn{3}{c}{ Sensors } \\
\cline { 1 - 2 } \cline { 5 - 7 } Parameter & Range & Step & & Type & Range & Resolution \\
\hline Ta & 24 days & $1 \mathrm{~s}$ & & Temp & $45^{\circ} \mathrm{C}$ & $0.044^{\circ} \pm 0.004^{\circ} \mathrm{C}$ \\
Ts & $18 \mathrm{~h}$ & $1 \mathrm{~s}$ & & Pressure & $300 \mathrm{psi}$ & $0.75 \pm 0.25 \mathrm{psi}$ \\
$\mathrm{Tw}$ & $34 \mathrm{~m}$ & $1 \mathrm{~s}$ & & Battery & $3.6 \mathrm{~V}$ & $3.45 \pm 0.15 \mathrm{mV}$ \\
\hline
\end{tabular}


steamed $\sim 4 \mathrm{~km}$ northeast of the free-drifting hydrophones for a first transmission test. The sound source was lowered from the stern of the Endeavor to a depth of approximately $15 \mathrm{~m}$ and activated manually six times, once per minute, each time emitting a linear frequency sweep ("pong") at maximum power from 260.5 to $263.5 \mathrm{~Hz}$ over $32 \mathrm{~s}$. This procedure was repeated at a second test site at a distance of $17 \mathrm{~km}$ in the same direction. We transmitted seven pongs at this second site. Unfortunately, one pod reset before deployment and another leaked, but the pods, at 10- and 40-m depth, respectively, performed as intended. Their acoustic recordings are summarized in Table 2. All transmitted signals were detected with distinct correlation peaks at both recording sites. The scatter in arrival times recorded at either location can be attributed to the manual activation of the sound source, since it could not be programmed to transmit on such a fast schedule. Similarly, the approximately 0.2 -s-longer travel times recorded by pod 2 are most likely due to the manual initialization of the clocks; that is, receiver 2 was initialized $0.2 \mathrm{~s}$ earlier than its counterpart. The strong correlation values recorded at either site are very encouraging and hint at a significantly larger signal propagation range than $17 \mathrm{~km}$.

To further validate the proper operation of the final tag design, we conducted one more field test with a few completely assembled tags south of the Mississippi delta in the Gulf of Mexico. The test was carried out following the same protocol applied during the previous field experiment. The tags were attached to the ends of two approximately 30-m-long ropes that were tied to two surface drifting buoys equipped with a GPS tracker. The sound source was then lowered repeatedly to a depth of approximately $25 \mathrm{~m}$ from the starboard side of the Endeavor at various distances starting at $\sim 130 \mathrm{~km}$. Two of the pods employed in the previous field test served as end-of-rope weights and reference data collectors. Figure 9 shows all signal paths between drifter and respective pong site.

The tags and pods recorded an almost-uniform water temperature near $27^{\circ} \mathrm{C}$ during the 40 -h test period. This provided for less-than-ideal acoustic conditions, since a warm surface layer tends to refract sound away from the surface. But a thin freshwater lens of Mississippi origin created a shallow surface sound channel such that transmissions could be detected as far as $60 \mathrm{~km}$ away. It is therefore not surprising that none of the tags and pods detected a signal emitted from any of the three farthest source sites (sites S1-S3 in Fig. 9) located $72-130 \mathrm{~km}$ away. The source pressure level, measured with a calibrated hydrophone, was $180 \mathrm{~dB}$ re $1 \mu \mathrm{Pa}$ at $1 \mathrm{~m}$.

Figure 10 shows the averages of all recorded signal propagation times as a function of sound source distance as deduced from GPS data. The inset in the bottom-right
TABLE 2. Acoustic data recorded during the field test off Long Island.

\begin{tabular}{lccccc}
\hline \hline \multicolumn{1}{c}{ Site } & Pong & \multicolumn{2}{c}{$\begin{array}{c}\text { Pod } 1(10-\mathrm{m} \\
\text { depth) Tp (s) } \\
\text { correlation }\end{array}$} & \multicolumn{2}{c}{$\begin{array}{c}\text { Pod 2 (40-m } \\
\text { depth) Tp (s) } \\
\text { correlation }\end{array}$} \\
\hline $14.8 \mathrm{~km}$ & 1 & 2.47 & 0.336 & 2.72 & 0.395 \\
& 2 & 3.41 & 0.289 & 3.66 & 0.340 \\
& 3 & 3.03 & 0.293 & 3.03 & 0.375 \\
& 4 & 3.38 & 0.363 & 3.56 & 0.402 \\
& 5 & 3.34 & 0.324 & 3.66 & 0.336 \\
Mean & 6 & 3.22 & 0.355 & 3.28 & 0.430 \\
Std dev & & 3.142 & 0.327 & 3.318 & 0.380 \\
& & 0.357 & 0.031 & 0.383 & 0.037 \\
& & & & & \\
& 1 & 10.94 & 0.293 & 11.03 & 0.199 \\
& 2 & 11.00 & 0.363 & 11.38 & 0.344 \\
& 3 & 11.56 & 0.301 & 11.66 & 0.313 \\
& 4 & 11.31 & 0.309 & 11.56 & 0.336 \\
& 5 & 10.56 & 0.352 & 10.84 & 0.329 \\
Mean & 6 & 11.50 & 0.305 & 11.81 & 0.406 \\
Std dev & 7 & 11.00 & 0.402 & 11.22 & 0.426 \\
\hline
\end{tabular}

corner lists GPS distance, number of detected signals, average signal propagation time, and standard deviation for all sites that yielded successful recordings.

Several interesting points can be made. First, the spread in signal propagation times is remarkably small, $0.053-0.132 \mathrm{~s}$. Excluding variations in the physical propagation path, this would translate to a distance resolution of better than $200 \mathrm{~m}$, which is not far from the theoretical best of $47 \mathrm{~m}$ resulting from the $32-\mathrm{Hz}$ sampling clock of the arrival time detector. Second, the apparent variations in sound speed partially stem from uncertainties in distance due to the 5-min resolution of the drifter GPS data (we did not interpolate). Third, it is strange that the best speed of sound to reconcile computed distance with measured travel time is not what might be expected on the basis of our estimates of surface/near-surface temperature and salinity ( $=1523 \mathrm{~m} \mathrm{~s}^{-1}$ for $25 \mathrm{psu}, 25^{\circ} \mathrm{C}, 20 \mathrm{~m}$ ) along the signal propagation path (Dushaw et al. 1993). Instead, the best fit for the data displayed in Fig. 10 is around $1490 \mathrm{~m} \mathrm{~s}^{-1}$ ( $\sim 2 \%$ less) with some uncertainty due to the paucity of data at the greater ranges. All travel times are relative to measured arrival times when the tags and pods were at $0-\mathrm{m}$ distance (placed inside the sound source on deck) immediately after retrieval.

\section{Summary}

We have described the development and validation of a new archival fish tag designed to track marine animals, whether in the water column (nekton) or on the bottom (benthic fish and crustaceans). The tag, similar in size to 


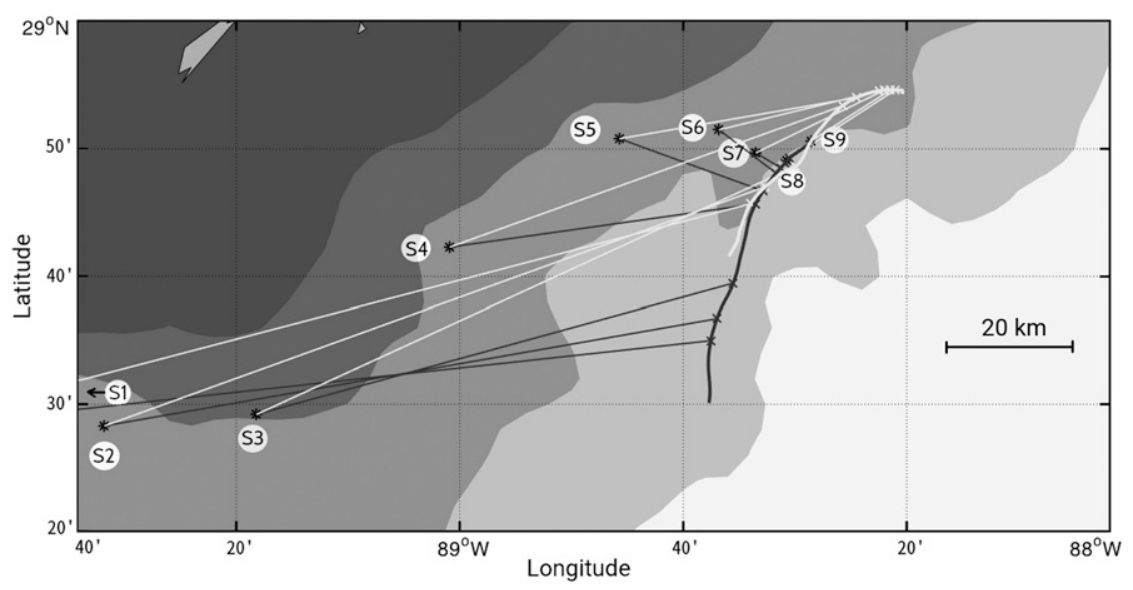

FIG. 9. Test site some $50 \mathrm{~km}$ southwest of the Mississippi delta. The plot shows the tracks of the two surface drifters (bold lines) and the acoustic signal paths from the various sound source locations (S1-S9) to the two drifter positions at the time of the sound transmissions (thin lines). The contours indicate the water depth in steps of $100 \mathrm{~m}$ (dark to light).

other archival tags, operates according to standard RAFOS tracking principles: it detects and records the arrival times of signals transmitted from moored sound sources. Navigational accuracy is determined by how well source and receiver clocks are aligned. Timing errors in the tag are kept quite small by compensating for the temperaturedependent drift of the crystal clock during the missions. Listening schedules can be set up according to tracking needs. Pressure and temperature can be sampled more frequently (up to 128 times) than searching for sound signatures to better resolve vertical movements and the diurnal behavior of the tagged species. Power to the fish tag comes from two button cell silver-oxide batteries, which enable an active tag lifetime of approximately 2 years.

Previous field studies involving archival tags have shown that tag return rates between $5 \%$ and $10 \%$ are possible depending on species and location. While this is encouraging, the need to retrieve the tag remains a major obstacle. If the presented technology is ported to larger, intermittently surfacing sampling platforms, however, it becomes possible to add a satellite transmitter that can upload the archived data at opportunistic

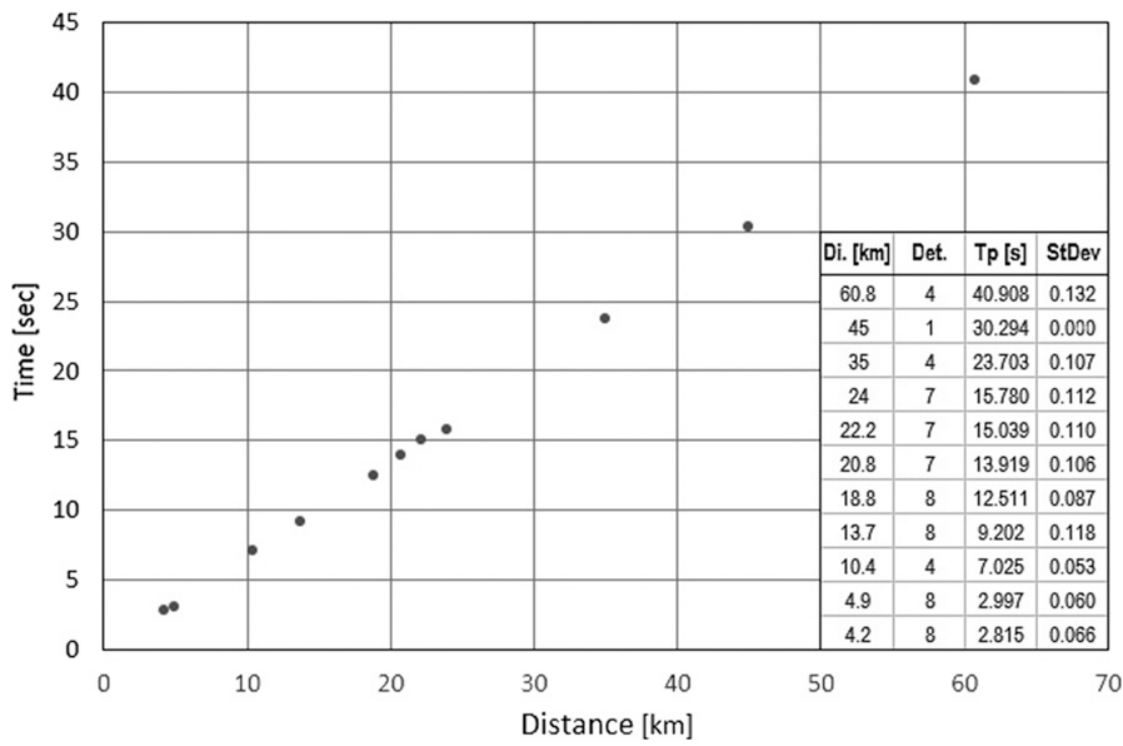

FIG. 10. Average signal propagation times vs distance as recorded by the tags and pods during the field test southwest of the Mississippi delta. (inset) The distance, the number of detections per location, the average propagation time, and the corresponding standard deviation. 
times. This feature and wireless device programming are the primary targets in future developments of this technology. As a first step in this direction, the fish chip technology is being ported to the next generation of RAFOS float, and it can be readily employed on gliders and autonomous underwater vehicles (AUVs) for interactive navigation. Since the fish chip requires virtually no power, it can also be added to long-range missions such as Argo floats, provided there is interest in tracking these with greater spatial resolution.

Acknowledgments. This work has been supported by subsequent NSF OTIC Awards 0326907, 1061083, and 1435698. The authors thank the following people for contributing in no minor way to the success of this endeavor: Tanya Wang for her skillful assembly of the final tags, Michael Platek for the assembly of the test pods, Bill Fanning for his technical support at sea, and the captain and the crew of the R/V Endeavor from which all field work was done. Funding by the Rhode Island Endeavor Program for two cruises is gratefully acknowledged.

\section{REFERENCES}

Blanchard, W. F., 1991: Air navigation systems Chapter 4. Hyperbolic airborne radio navigation aids-A navigator's view of their history and development. J. Navig., 44, 285-315, https://doi.org/10.1017/S0373463300010092.

Block, B. A., and Coauthors, 2001: Migratory movements, depth preferences, and thermal biology of Atlantic bluefin tuna. Science, 293, 1310-1314, https://doi.org/10.1126/science.1061197.

Bower, A. S., L. Armi, and I. Ambar, 1995: Direct evidence of meddy formation off the southwestern coast of Portugal. Deep-Sea Res. I, 42, 1621-1630, https://doi.org/10.1016/ 0967-0637(95)00045-8.

Crols, J., and M. Steyaert, 1994: Switched-opamp: An approach to realize full CMOS switched-capacitor circuits at very low power supply voltages. IEEE J. Solid-State Circuits, 29, 936-942, https://doi.org/10.1109/4.297698.

Dushaw, B. D., P. R. Worcester, B. D. Cornuelle, and B. M. Howe, 1993: On equations for the speed of sound in seawater. J. Acoust. Soc. Amer., 93, 255-275, https://doi.org/10.1121/ 1.405660 .

Eriksson, H., P. O. Börjesson, P. Oding, and N.-G. Holmer, 1994: A robust correlation receiver for distance estimation. IEEE Trans. Ultrason. Ferroelectr. Freq. Control, 41, 596-603, https://doi.org/10.1109/58.308494.

Fischer, G., and F. Luo, 2012: Jitter in ultra-low power audio-range PLLs. Proc. IEEE 55th Int. Midwest Symp. on Circuits and Sysems (MWSCAS), Boise, ID, IEEE, 550-553, https:// doi.org/10.1109/mwscas.2012.6292079

_, S. Lee, M. Obara, P. Kasturi, H. T. Rossby, and C. W. Recksiek, 2006: Tracking fishes with a microwatt acoustic receiver-An archival tag development. IEEE
J. Oceanic Eng., 31, 975-985, https://doi.org/10.1109/ JOE.2006.880376.

Gazit, T., R. Apostle, and R. Branton, 2013: Deployment, tracking, and data management: Technology and science for a global ocean tracking network. J. Int. Wildl. Law Policy, 16, 112-127, https://doi.org/10.1080/ 13880292.2013.805058.

Gunn, J., and J. Hartog, 1999: Archival tag project report. CRIMP Southern Bluefin Tuna Recruitment Monitoring and Tagging Program Workshop Rep. 11, 5 pp.

Harrison, C. H., 1996: Formulas for ambient noise level and coherence. J. Acoust. Soc. Amer., 99, 2055-2066, https:// doi.org/10.1121/1.415392.

Metcalfe, J. D., G. P. Arnold, and B. H. Holford, 1994: The migratory behavior of plaice in the North Sea as revealed by data storage tags. Proc. ICES Council Meeting 1994/Mini 11, International Council for the Exploration of the Sea, Plymouth, United Kingdom, 13 pp.

Parvin, S. J., J. R. Nedwell, and E. Harland, 2007: Lethal and physical injury of marine mammals, and requirements for Passive Acoustic Monitoring. Subacoustech Ltd. Rep. 565R0212, 38 pp.

Rossby, H. T., 1996: The North Atlantic current and surrounding waters: At the crossroads. Rev. Geophys., 34, 463-481, https:// doi.org/10.1029/96RG02214.

_ - 2007: Evolution of Lagrangian methods in oceanography. Lagrangian Analysis and Prediction of Coastal and Ocean Dynamics, A. Griffa et al., Eds., Cambridge University Press, 1-38, https://doi.org/10.1017/ CBO9780511535901.002.

_ A. Voorhis, and D. Webb, 1975: A quasi-Lagrangian study of mid-ocean variability using long-range SOFAR floats. J. Mar. Res., 33, 355-382.

_ J. Atmos. Oceanic Technol., 3, 672-679, https://doi.org/ 10.1175/1520-0426(1986)003<0672:TRS $>2.0 . \mathrm{CO} ; 2$.

—_, J. Ellis, and D. C. Webb, 1993: An efficient sound source for wide-area RAFOS navigation. J. Atmos. Oceanic Technol., 10, 397-403, https://doi.org/10.1175/ 1520-0426(1993)010<0397:AESSFW>2.0.CO;2.

Schneider, M. C., and C. Galup-Montoro, 2010: Fundamentals of sampled-data circuits. CMOS Analog Design Using AllRegion MOSFET Modeling, Cambridge University Press, 404-451, https://doi.org/10.1017/CBO9780511803840.011.

Urick, R. J., 1983: Principles of Underwater Sound. Peninsula Publishing, 423 pp.

— 1984: Ambient noise in the sea. Department of the Navy Naval Systems Command Undersea Warfare Technology Office Rep. OEC 20070117128, 194 pp.

Vittoz, E. A., and O. Neyroud, 1979: A lowvoltage CMOS bandgap reference. IEEE J. SolidState Circuits, 14, 573-577, https://doi.org/10.1109/ JSSC.1979.1051218.

Weber, D., 2009: VEMCO acoustic telemetry-New user guide. AMIRIX Systems Inc. Publ. DOC-004934-01, $22 \mathrm{pp}$.

Wenz, G. M., 1962: Acoustic ambient noise in the ocean. J. Acoust. Soc. Amer., 34, 1936, https://doi.org/10.1121/1.1909155. 